\title{
Maximum Simulated Likelihood Estimation: Techniques and Applications in Economics
}

\author{
Ivan Jeliazkov and Alicia Lloro
}

\begin{abstract}
This chapter discusses maximum simulated likelihood estimation when construction of the likelihood function is carried out by recently proposed Markov chain Monte Carlo (MCMC) methods. The techniques are applicable to parameter estimation and Bayesian and frequentist model choice in a large class of multivariate econometric models for binary, ordinal, count, and censored data. We implement the methodology in a study of the joint behavior of four categories of U.S. technology patents using a copula model for multivariate count data. The results reveal interesting complementarities among several patent categories and support the case for joint modeling and estimation. Additionally, we find that the simulated likelihood algorithm performs well. Even with few MCMC draws, the precision of the likelihood estimate is sufficient for producing reliable parameter estimates and carrying out hypothesis tests.
\end{abstract}

\section{Introduction}

The econometric analysis of models for multivariate discrete data is often complicated by intractability of the likelihood function, which can rarely be evaluated directly and typically has to be estimated by simulation. In such settings, the efficiency of likelihood estimation plays a key role in determining the theoretical properties and practical appeal of standard optimization algorithms that rely on those estimates. For this reason, the development of fast and statistically efficient techniques for estimating the value of the likelihood function has been at the forefront of much of the research on maximum simulated likelihood estimation in econometrics.

In this paper we examine the performance of a method for estimating the ordinate of the likelihood function which was recently proposed in [8]. The method is

Department of Economics, University of California, Irvine, 3151 Social Science Plaza, Irvine, CA 92697, e-mail: ivan@uci.edu and alloro@uci.edu. 
rooted in Markov chain Monte Carlo (MCMC) theory and simulation [3, 4, 15, 18], and its ingredients have played a central role in Bayesian inference in econometrics and statistics. The current implementation of those methods, however, is intended to examine their applicability to purely frequentist problems such as maximum likelihood estimation and hypothesis testing.

We implement the methodology to study firm-level patent registration data in four patent categories in the "computers \& instruments" industry during the 1980s. One goal of this application is to examine how patent counts in each category are affected by firm characteristics such as sales, workforce size, and research \& development (R\&D) capital. A second goal is to study the degree of complementarity or substitutability that emerges among patent categories due to a variety of unobserved factors, such as firms' internal R\&D decisions, resource concentration, managerial dynamics, technological spillovers, and the relevance of innovations across category boundaries. These factors can affect multiple patent categories simultaneously and necessitate the specification of a joint empirical structure that can flexibly capture interdependence patterns.

We approach these tasks by considering a copula model for multivariate count data which enables us to pursue joint modeling and estimation. Because the outcome probabilities in the copula model are difficult to evaluate, we rely on MCMC simulation to evaluate the likelihood function. Moreover, to improve the performance of the optimization algorithm, we implement a quasi-Newton optimization method due to [1] that exploits a fundamental statistical relation to avoid direct computation of the Hessian matrix of the log-likelihood function. The application demonstrates that the simulated likelihood algorithm performs very well - even with few MCMC draws, the precision of the likelihood estimate is sufficient for producing reliable parameter estimates and hypothesis tests. The results support the case for joint modeling and estimation in our application and reveal interesting complementarities among several patent categories.

The remainder of this chapter is organized as follows. In Section 2, we present the copula model that we use in our application and the likelihood function that we use in estimation. The likelihood function is difficult to evaluate because it is given by a set of integrals with no closed-form solution. For this reason, in Section 3, we present the MCMC-based simulation algorithm for evaluating this function and discuss how it can be embedded in a standard optimization algorithm to maximize the $\log$-likelihood function and yield parameter estimates and standard errors. Section 4 presents the results from our patent application and demonstrates the performance of the estimation algorithm. Section 5 offers concluding remarks.

\section{Copula Model}

In analyzing multiple data series, it is typically desirable to pursue joint modeling and estimation. Doing so allows researchers to investigate dependence structures among the individual variables of interest, leads to gains in estimation efficiency, and 
is also important for mitigating misspecification problems in nonlinear models. In many applications, however, a suitable joint distribution may be unavailable or difficult to specify. This problem is particularly prevalent in multivariate discrete data settings, and in cases where the variables are of different types (e.g. some continuous, some discrete or censored). One area of research where incorporating a flexible and interpretable correlation structure has been difficult is the empirical analysis of multivariate count data $[2,21]$. As a consequence, models for multivariate counts have typically sacrificed generality for the sake of retaining computational tractability. To deal with the aforementioned difficulties, we resort to a copula modeling approach whose origins can be traced back to [17].

Formally, a copula maps the unit hypercube $[0,1]^{q}$ to the unit interval $[0,1]$ and satisfies the following conditions:

1. $C\left(1, \ldots, 1, a_{p}, 1, \ldots, 1\right)=a_{p}$ for every $p \in\{1, \ldots, q\}$ and all $a_{p} \in[0,1]$;

2. $C\left(a_{1}, \ldots, a_{q}\right)=0$ if $a_{p}=0$ for any $p \in\{1, \ldots, q\}$;

3. $C$ is $q$-increasing, i.e. any hyperrectangle in $[0,1]^{q}$ has non-negative $C$-volume.

The generality of the approach rests on the recognition that a copula can be viewed as a $q$-dimensional distribution function with uniform marginals, each of which can be related to an arbitrary known cumulative distribution function (cdf) $F_{j}(\cdot)$, $j=1, \ldots, q$. For example, if a random variable $u_{j}$ is uniform $u_{j} \sim U(0,1)$, and $y_{j}=F_{j}^{-1}\left(u_{j}\right)$, then it is easy to show that $y_{j} \sim F_{j}(\cdot)$. As a consequence, if the variables $y_{1}, \ldots, y_{q}$ have corresponding univariate cdfs $F_{1}\left(y_{1}\right), \ldots, F_{q}\left(y_{q}\right)$ taking values in $[0,1]$, a copula is a function that can be used to link or "couple" those univariate marginal distributions to produce the joint distribution function $F\left(y_{1}, \ldots, y_{q}\right)$ :

$$
F\left(y_{1}, \ldots, y_{q}\right)=C\left(F_{1}\left(y_{1}\right), \ldots, F_{q}\left(y_{q}\right)\right) .
$$

A detailed overview of copulas is provided in [9], [13], and [20]. The key feature that will be of interest here is that they provide a way to model dependence among multiple random variables when their joint distribution is not easy to specify, including cases where the marginal distributions $\left\{F_{j}(\cdot)\right\}$ belong to entirely different parametric classes.

There are several families of copulas, but the Gaussian copula is a natural modeling choice when one is interested in extensions beyond the bivariate case. The Gaussian copula is given by

$$
C(u \mid \Omega)=\Phi_{q}\left(\Phi^{-1}\left(u_{1}\right), \ldots, \Phi^{-1}\left(u_{q}\right) \mid \Omega\right),
$$

where $u=\left(u_{1}, \ldots, u_{q}\right)^{\prime}, \Phi$ represents the standard normal cdf, and $\Phi_{q}$ is the cdf for a multivariate normal vector $z=\left(z_{1}, \ldots, z_{q}\right)^{\prime}, z \sim N(0, \Omega)$, where $\Omega$ is in correlation form with ones on the main diagonal. The data generating process implied by the Gaussian copula specification is given by

$$
y_{i j}=F_{i j}^{-1}\left\{\Phi\left(z_{i j}\right)\right\}, \quad z_{i} \sim N(0, \Omega), \quad i=1, \ldots, n, \quad j=1, \ldots, q,
$$


where $F_{i j}$ is a cdf specified in terms of a vector of parameters $\theta_{j}$ and covariates $x_{i j}, q$ is the dimension of each vector $y_{i}=\left(y_{i 1}, \ldots, y_{i q}\right)^{\prime}$, and $n$ is the sample size. Note that the correlation matrix $\Omega$ for the latent $z_{i}$ induces dependence among the elements of $y_{i}$ and that the copula density will typically be analytically intractable.

The structures in (1), (2), and (3) are quite general and apply to both discrete and continuous outcomes. However, it is important to recognize that the inverse cdf mapping $F_{i j}^{-1}(\cdot)$ in (3) is one-to-one when $y_{i j}$ is continuous and many-to-one when $y_{i j}$ is discrete. Therefore, in the latter case it is necessary to integrate over the values of $z_{i}$ that lead to the observed $y_{i}$ in order to obtain their joint distribution. In our implementation, this integration is performed by MCMC methods.

In this chapter, we use the Gaussian copula framework to specify a joint model for multivariate count data, where each count variable $y_{i j} \in\{0,1,2, \ldots\}$ follows a variable-specific negative binomial distribution

$$
y_{i j} \sim N B\left(\lambda_{i j}, \alpha_{j}\right),
$$

with probability mass function (pmf) given by

$$
\operatorname{Pr}\left(y_{i j} \mid \lambda_{i j}, \alpha_{j}\right)=\frac{\Gamma\left(\alpha_{j}+y_{i j}\right) r_{i j}^{\alpha_{j}}\left(1-r_{i j}\right)^{y_{i j}}}{\Gamma\left(1+y_{i j}\right) \Gamma\left(\alpha_{j}\right)}, \quad \lambda_{i j}>0, \quad \alpha_{j}>0,
$$

where $r_{i j}=\alpha_{j} /\left(\alpha_{j}+\lambda_{i j}\right)$, and dependence on the covariates is modeled through $\lambda_{i j}=\exp \left(x_{i j}^{\prime} \beta_{j}\right)$. Here, and in the remainder of this chapter, all vectors will be taken to be column vectors. The distribution in (4) has mean $\lambda_{i j}$ and variance $\lambda_{i j}\left(1+\lambda_{i j} / \alpha_{j}\right)$, so that, depending on $\alpha_{j}$, it allows for varying degrees of overdispersion. The variance can be much larger than the mean for small values of $\alpha_{j}$, but in the limit (as $\alpha_{j} \rightarrow \infty$ ) the two are equal, as in the Poisson model where the conditional variance equals the conditional mean. Negative binomial models are carefully reviewed in [2], [6], and [21].

The cdf for the negative binomial distribution is obtained by summing the pmf in (4) for values less than or equal to $y_{i j}$ :

$$
F_{j}\left(y_{i j} \mid \lambda_{i j}, \alpha_{j}\right)=\sum_{k=0}^{y_{i j}} \operatorname{Pr}\left(k \mid \lambda_{i j}, \alpha_{j}\right)
$$

To relate the negative binomial distribution to the Gaussian copula, the pmf and cdf computed in (4) and (5), respectively, can be used to find unique, recursively determined cutpoints

$$
\begin{aligned}
& \gamma_{i j, U}=\Phi^{-1}\left(F_{j}\left(y_{i j} \mid \beta_{j}, \alpha_{j}\right)\right) \\
& \gamma_{i j, L}=\Phi^{-1}\left(F_{j}\left(y_{i j} \mid \beta_{j}, \alpha_{j}\right)-\operatorname{Pr}\left(y_{i j} \mid \lambda_{i j}, \alpha_{j}\right)\right)
\end{aligned}
$$

that partition the standard normal distribution so that for $z_{i j} \sim N(0,1)$, we have $\operatorname{Pr}\left(z_{i j} \leq \gamma_{i j, U}\right)=F_{j}\left(y_{i j} \mid \lambda_{i j}, \alpha_{j}\right)$ and $\operatorname{Pr}\left(\gamma_{i j, L}<z_{i j} \leq \gamma_{i j, U}\right)=\operatorname{Pr}\left(y_{i j} \mid \lambda_{i j}, \alpha_{j}\right)$. Hence, the cutpoints in (6) provide the range $B_{i j}=\left(\gamma_{i j, L}, \gamma_{i j, U}\right]$ of $z_{i j}$ that is consistent with 


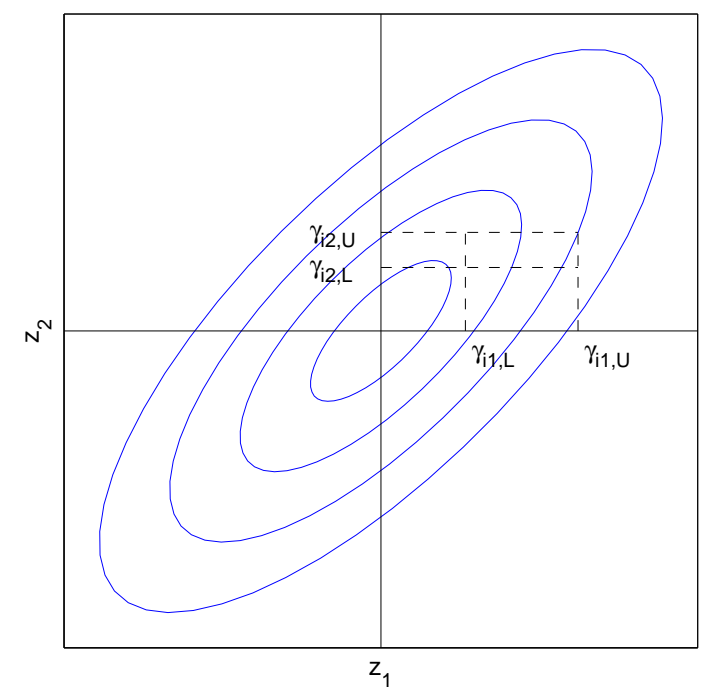

Fig. 1 An example of the region of integration implied by a bivariate Gaussian copula model

each observed outcome $y_{i j}$ in (3). In turn, because $z_{i}=\left(z_{i 1}, \ldots, z_{i q}\right)^{\prime} \sim N(0, \Omega)$, the Gaussian copula representation implies that the joint probability of observing the vector $y_{i}=\left(y_{i 1}, \ldots, y_{i q}\right)^{\prime}$ is given by

$$
\operatorname{Pr}\left(y_{i} \mid \theta, \Omega\right)=\int_{B_{i q}} \cdots \int_{B_{i 1}} f_{N}\left(z_{i} \mid 0, \Omega\right) d z_{i},
$$

in which $f_{N}(\cdot)$ denotes the normal density and, for notational convenience, we let $\theta=\left(\theta_{1}^{\prime}, \ldots, \theta_{q}^{\prime}\right)^{\prime}$, where $\theta_{j}=\left(\beta_{j}^{\prime}, \alpha_{j}\right)^{\prime}$ represents the parameters of the $j$ th marginal model, which determine the regions of integration $B_{i j}=\left(\gamma_{i j, L}, \gamma_{i j, U}\right], j=1, \ldots, q$. Figure 1 offers an example of how the region of integration is constructed in the simple bivariate case. Because of the dependence introduced by the correlation matrix $\Omega$, the probabilities in (7) have no closed-form solution and will be estimated by MCMC simulation methods in this chapter. Once computed, the probabilities in (7), also called likelihood contributions, can be used to construct the likelihood function

$$
f(y \mid \theta, \Omega)=\prod_{i=1}^{n} \operatorname{Pr}\left(y_{i} \mid \theta, \Omega\right) .
$$

The likelihood function is then used in obtaining maximum likelihood estimates $\hat{\theta}$ and $\hat{\Omega}$, standard errors, and in performing model comparisons and hypothesis tests. Because the likelihood contributions are obtained by simulation, $f(y \mid \theta, \Omega)$ in (8) is referred to as the simulated likelihood function, and the estimates $\hat{\theta}$ and $\hat{\Omega}$ are called maximum simulated likelihood estimates. We next discuss the simulation and optimization techniques that are used to obtain those estimates. 


\section{Estimation Methodology}

Estimation by maximum simulated likelihood requires evaluation of the outcome probabilities for each observation vector $y_{i}$. Because each outcome probability in (7) is defined by an analytically intractable multidimensional integral, in Section 3.1 we describe a method for evaluating the outcome probabilities which was introduced in [8]. The method, called the Chib-Ritter-Tanner (CRT) method, stems from developments in MCMC simulation and Bayesian model choice and is well suited for evaluating outcome probabilities that comprise the likelihood of a variety of discrete data models. Because the CRT estimator produces continuous and differentiable estimates of (8), in Section 3.2 we describe how it can be applied in standard quasiNewton gradient-based optimization using the Berndt-Hall-Hall-Hausman (BHHH) approach proposed in [1]. The BHHH approach exploits a fundamental statistical relation to avoid direct computation of the Hessian matrix of the log-likelihood function in the optimization algorithm.

\subsection{The CRT Method}

The CRT method, proposed in [8], is derived from theory and techniques in MCMC simulation and Bayesian model selection (see [3], [15]), where evaluation of multidimensional integrals with no analytical solution is routinely required. To understand the approach, note that the outcome probability in (7) can be rewritten as

$$
\operatorname{Pr}\left(y_{i} \mid \theta, \Omega\right)=\int 1\left\{z_{i} \in B_{i}\right\} f_{N}\left(z_{i} \mid 0, \Omega\right) d z_{i}=\frac{1\left\{z_{i} \in B_{i}\right\} f_{N}\left(z_{i} \mid 0, \Omega\right)}{f_{T N_{B_{i}}}\left(z_{i} \mid 0, \Omega\right)}
$$

where $B_{i}=B_{i 1} \times B_{i 2} \times \cdots \times B_{i q}$ and $f_{T N_{B_{i}}}(\cdot)$ represents the truncated normal density that accounts for the truncation constraints reflected in $B_{i}$. This representation follows by Bayes formula because $\operatorname{Pr}\left(y_{i} \mid \theta, \Omega\right)$ is the normalizing constant of a truncated normal distribution, and its representation in terms of the quantities in (9) is useful for developing an estimation strategy that is simple and efficient. As discussed in [3], this identity is particularly useful because it holds for any value of $z_{i} \in B_{i}$ and therefore, given that the numerator quantities $1\left\{z_{i}^{*} \in B_{i}\right\}$ and $f_{N}\left(z_{i}^{*} \mid 0, \Omega\right)$ in (9) are directly available, the calculation is reduced to finding an estimate of the ordinate $f_{T N_{B_{i}}}\left(z_{i}^{*} \mid 0, \Omega\right)$ at a single point $z_{i}^{*} \in B_{i}$, typically taken to be the sample mean of the draws $z_{i} \sim T N_{B_{i}}(0, \Omega)$ that will be simulated in the estimation procedure (details will be presented shortly). The log-probability is subsequently obtained as

$$
\ln \widehat{\operatorname{Pr}}\left(y_{i} \mid \theta, \Omega\right)=\ln f_{N}\left(z_{i}^{*} \mid 0, \Omega\right)-\ln \widehat{f_{T N_{B_{i}}}}\left(z_{i}^{*} \mid 0, \Omega\right),
$$

To estimate $\widehat{f_{T N_{B_{i}}}}\left(z_{i}^{*} \mid 0, \Omega\right)$ in (10), the CRT method relies on random draws $z_{i} \sim$ $T N_{B_{i}}(0, \Omega)$ which are produced by the Gibbs sampling algorithms of [5] or [16], where a new value for $z_{i}$ is generated by iteratively simulating each element $z_{i j}$ 
from its full-conditional density $z_{i j} \sim f\left(z_{i j} \mid y_{i j},\left\{z_{i k}\right\}_{k \neq j}, \Omega\right)=T N_{B_{i j}}\left(\mu_{i j}, \sigma_{i j}^{2}\right)$ for $j=1, \ldots, q$. In the preceding, $\mu_{i j}$ and $\sigma_{i j}^{2}$ are the conditional mean and variance of $z_{i j}$ given $\left\{z_{i k}\right\}_{k \neq j}$ and are obtained by the usual formulas for a conditional Gaussian density. MCMC simulation of $z_{i} \sim T N_{B_{i}}(0, \Omega)$ is an important tool for drawing from this density, which is non-standard due to the multiple constraints defining the set $B_{i}$ and the correlations in $\Omega$.

The Gibbs transition kernel for moving from a point $z_{i}$ to $z_{i}^{*}$ is given by the product of univariate truncated normal full-conditional densities

$$
K\left(z_{i}, z_{i}^{*} \mid y_{i}, \theta, \Omega\right)=\prod_{j=1}^{J} f\left(z_{i j}^{*} \mid y_{i},\left\{z_{i k}^{*}\right\}_{k<j},\left\{z_{i k}\right\}_{k>j}, \theta, \Omega\right) .
$$

Because the full-conditional densities represent the fundamental building blocks of the Gibbs sampler, the additional coding involved in evaluating (11) is minimized. By virtue of the fact that the Gibbs sampler satisfies Markov chain invariance (see $[18,4])$, in our context we have that

$$
f_{T N_{B_{i}}}\left(z_{i}^{*} \mid 0, \Omega\right)=\int K\left(z_{i}, z_{i}^{*} \mid y_{i}, \theta, \Omega\right) f_{T N_{B_{i}}}\left(z_{i} \mid 0, \Omega\right) d z_{i},
$$

a more general version of which was exploited for density estimation in [15]. Therefore, an estimate of $f_{T N_{B_{i}}}\left(z_{i}^{*} \mid 0, \Omega\right)$ for use in (9) or (10) can be obtained by invoking (12) and averaging the transition kernel $K\left(z_{i}, z_{i}^{*} \mid y_{i}, \theta, \Omega\right)$ with respect to draws from the truncated normal distribution $z_{i}^{(g)} \sim T N_{B_{i}}(0, \Omega), g=1, \ldots, G$, i.e.

$$
\widehat{f_{T N_{B_{i}}}}\left(z_{i}^{*} \mid 0, \Omega\right)=\frac{1}{G} \sum_{g=1}^{G} K\left(z_{i}^{(g)}, z_{i}^{*} \mid y_{i}, \theta, \Omega\right) \text {. }
$$

When repeated evaluation of (12) is required, e.g. in evaluating derivatives of $f(y \mid \theta, \Omega)$, one should remember to reset the random number generation seed used in the simulations. The CRT method produces continuous and differentiable estimates of $\operatorname{Pr}\left(y_{i} \mid \theta, \Omega\right)$ and can thus be applied directly in derivative-based optimization as discussed next.

\subsection{Optimization Technique}

Let $\psi$ represent the vector of parameters that enter the log-likelihood function $\ln f(y \mid \psi)$. For the copula model that we considered in Section 2, $\psi$ consists of the elements of $\theta$ and the unique off-diagonal entries of $\Omega$ (recall that $\Omega$ is symmetric positive definite matrix with ones on the main diagonal) and the likelihood function $f(y \mid \psi)$ is given in (8). Standard Newton-Raphson maximization of the loglikelihood function $\ln f(y \mid \psi)$ proceeds by updating the value of the parameter vector in iteration $t, \psi_{t}$, to a new value, $\psi_{t+1}$, using the formula 


$$
\psi_{t+1}=\psi_{t}-\lambda H_{t}^{-1} g_{t},
$$

where $g_{t}=\partial \ln f\left(y \mid \psi_{t}\right) / \partial \psi_{t}$ and $H_{t}=\partial^{2} \ln f\left(y \mid \psi_{t}\right) / \partial \psi_{t} \partial \psi_{t}^{\prime}$ are the gradient vector and Hessian matrix, respectively, of the log-likelihood function at $\psi_{t}$ and $\lambda$ is a step size. Gradient-based methods are widely used in log-likelihood optimization because many statistical models have well-behaved log-likelihood functions and gradients and Hessian matrices are often required for statistical inference, e.g. in obtaining standard errors or Lagrange multiplier test statistics. The standard NewtonRaphson method, however, has well-known drawbacks. One is that computation of the Hessian matrix can be quite computationally intensive. For a $k$ dimensional parameter vector $\psi$, computing the Hessian requires $O\left(k^{2}\right)$ evaluations of the loglikelihood function. In the context of simulated likelihood estimation, where $k$ can be very large and each likelihood evaluation can be very costly, evaluation of the Hessian presents a significant burden that adversely affects the computational efficiency of Newton-Raphson. Another problem is that $(-H)$ may fail to be positive definite. This may be due to purely numerical issues (e.g. the computed Hessian may be a poor approximation to the analytical one) or it may be caused by non-concavity of the log-likelihood function. In those instances, the Newton-Raphson iterations will fail to converge to a local maximum.

To deal with these difficulties, [1] noted that an application of a fundamental statistical relationship, known as the information identity, obviates the need for direct computation of the Hessian. Because we are interested in maximizing a statistical function given by the sum of the log-likelihood contributions over a sample of observations, it is possible to use statistical theory to speed up the iterations. In particular, by definition we have

$$
\int f(y \mid \psi) d y=1
$$

where it is assumed that if there are any limits of integration, they do not depend on the parameters $\psi$. With this assumption, an application of Leibniz's theorem implies that $\partial\left\{\int f(y \mid \psi) d y\right\} / \partial \psi=\int \partial f(y \mid \psi) / \partial \psi d y$. Moreover, because $\partial f(y \mid \psi) / \partial \psi=$ $\{\partial \ln f(y \mid \psi) / \partial \psi\} f(y \mid \psi)$, upon differentiation of both sides of (15) with appropriate substitutions, we obtain

$$
\int \frac{\partial \ln f(y \mid \psi)}{\partial \psi} f(y \mid \psi) d y=0 .
$$

Differentiating (16) with respect to $\psi$ once again (recalling that under our assumptions we can interchange integration and differentiation), we get

$$
\int\left\{\frac{\partial^{2} \ln f(y \mid \psi)}{\partial \psi \partial \psi^{\prime}} f(y \mid \psi)+\frac{\partial \ln f(y \mid \psi)}{\partial \psi} \frac{\partial f(y \mid \psi)}{\partial \psi^{\prime}}\right\} d y=0,
$$

where, taking advantage of the equality $\partial f(y \mid \psi) / \partial \psi=\{\partial \ln f(y \mid \psi) / \partial \psi\} f(y \mid \psi)$ once again, we obtain the primary theoretical result underlying the BHHH approach 


$$
-\int \frac{\partial^{2} \ln f(y \mid \psi)}{\partial \psi \partial \psi^{\prime}} f(y \mid \psi) d y=\int \frac{\partial \ln f(y \mid \psi)}{\partial \psi} \frac{\partial \ln f(y \mid \psi)}{\partial \psi^{\prime}} f(y \mid \psi) d y .
$$

The left side of equation (17) gives $E(-H)$, whereas on the right side we have $E\left(g g^{\prime}\right)$ which also happens to be $\operatorname{Var}(g)$ because from (16) we know that $E(g)=0$. Now, because the log-likelihood is the sum of independent log-likelihood contributions, i.e. $\ln f(y \mid \psi)=\sum_{i=1}^{n} \ln f\left(y_{i} \mid \psi\right)$, it follows that

$$
\operatorname{Var}(g)=\sum_{i=1}^{n} \operatorname{Var}\left(g_{i}\right) \approx \sum_{i=1}^{n} g_{i} g_{i}^{\prime},
$$

in which $g_{i}=\partial \ln f\left(y_{i} \mid \psi\right) / \partial \psi$. Therefore, the BHHH algorithm for maximizing the log-likelihood function relies on the recursions

$$
\psi_{t+1}=\psi_{t}+\lambda B_{t}^{-1} g_{t}
$$

where $B_{t}=\sum_{i=1}^{n}\left[\frac{\partial \ln f\left(y_{i} \mid \psi_{t}\right)}{\partial \psi_{t}}\right]\left[\frac{\partial \ln f\left(y_{i} \mid \psi_{t}\right)}{\partial \psi_{t}}\right]^{\prime}$ is used in lieu of $-H_{t}$ in (14).

Working with the outer product of gradients matrix, $B_{t}$, has several important advantages. First, computation of the gradients requires $O(k)$ likelihood evaluations and hence yields significant computational benefits relative to direct evaluation of $H_{t}$ which requires $O\left(k^{2}\right)$ such evaluations. Note that $\left\{\partial \ln f\left(y_{i} \mid \psi_{t}\right) / \partial \psi_{t}\right\}$ are calculated anyway in computing $g_{t}$ and that obtaining $B_{t}$ only involves taking their outer product but requires no further evaluations of $\ln f(y \mid \psi)$. Second, $B_{t}$ is necessarily positive definite, as long as the parameters are identified, even in regions where the log-likelihood is convex. Hence, the BHHH algorithm guarantees an increase in $\ln f(y \mid \psi)$ for a small enough step size $\lambda$. Finally, $B_{t}$ is typically more computationally stable than $H_{t}$, thereby reducing numerical difficulties in practice (e.g. with inversion, matrix decomposition, etc.).

We make an important final remark about the interplay between simulation and optimization in maximum simulated likelihood estimation: precise estimation of the $\log$-likelihood is essential for correct statistical inference. Specifically, it is crucial for computing likelihood ratio statistics, information criteria, marginal likelihoods and Bayes factors, and is also key to mitigating simulation biases in the maximum simulated likelihood estimation of parameters, standard errors, and confidence intervals (see [12], [19]). For instance, if the probabilities that enter $f(y \mid \psi)$ are estimated imprecisely, the maximum likelihood estimate will be biased (by Jensen's inequality) and the estimates of $\left\{\partial \ln f\left(y_{i} \mid \psi_{t}\right) / \partial \psi_{t}\right\}$ will be dominated by simulation noise. This adversely affects the estimated standard errors because $B_{t}$ is inflated by simulation noise rather than capturing genuine log-likelihood curvature. Hence, relying on the modal value of $B_{t}^{-1}$ as an estimate of the covariance matrix of $\hat{\psi}$ will produce standard errors and confidence bands that are too optimistic (too small). In extreme cases, parameters that are weakly identified may appear to be estimated well, due entirely to the simulation noise. Such problems can be recognized by examining the behavior of the estimated standard errors (square root of the diagonal of the modal 
Table 1 Descriptive statistics for the explanatory variables in the patent count application

\begin{tabular}{llrc}
\hline \hline Variable & Description & Mean & SD \\
\hline $\ln (S A L E S)$ & Log of real sales (millions) & 6.830 & 1.703 \\
$\ln (W F)$ & Log of number of company employees & 2.398 & 1.717 \\
$\ln (R D C)$ & Log of real R\&D capital (millions) & 5.593 & 1.815 \\
\hline
\end{tabular}

value of $B_{t}^{-1}$ ) for different values of the simulation size $G$ in (13) to determine whether they are stable or tend to decrease as $G$ is increased.

\section{Application}

In this section, we implement the methodology developed earlier to study the joint behavior of firm-level patent registrations in four technology categories in the "computers \& instruments" industry during the 1980s. We use the data sample of [10], which consists of $n=498$ observations on 254 manufacturing firms from the U.S. Patent $\&$ Trademark Office data set discussed in [7] and [11]. The response variable is a $4 \times 1$ vector $y_{i}(i=1, \ldots, 498)$ containing firm-level counts of registered patents in communications (COM), computer hardware \& software (CHS), computer peripherals $(\mathrm{CP})$, and information storage (IS). The explanatory variables reflect the characteristics of individual firms and, in addition to a category specific intercept, include sales (SALES), workforce size (WF), and R\&D capital (RDC). Sales are measured by the annual sales revenue of each firm, while the size of the workforce is given by the number of employees that the firm reports to stockholders. R\&D capital is a variable constructed from the history of R\&D investment using inventory and depreciation rate accounting standards discussed in [7]. All explanatory variables, except the intercept, are measured on the logarithmic scale. Table 1 contains variable explanations along with descriptive statistics.

To analyze these multivariate counts, we use a Gaussian copula model with negative binomial marginals which was presented in Section 2. The negative binomial specification is suitable for this application because patent counts exhibit a heavy right tail, and hence it is useful to specify a model that can account for the possible presence and extent of over-dispersion. In addition to examining how patents in each category are affected by firm characteristics, joint modeling allows us to study the interdependence of patent counts that emerges due to technological spillovers, managerial incentives, and internal R\&D decisions. For instance, technological breakthroughs and know-how in one area may produce positive externalities and spill over to other areas. Moreover, significant discoveries may produce patents in multiple categories, resulting in positive correlation among patent counts. Alternatively, the advancement of a particular technology may cause a firm to re-focus and concentrate its resources to that area at the expense of others, thereby producing negative correlations. The dependence structure embodied in the correlation matrix $\Omega$ of 
the Gaussian copula model that we consider is intended to capture these and other factors that can affect multiple patent categories simultaneously.

We estimate the copula model by first estimating the parameters of each negative binomial model separately by maximum likelihood and then using those estimates as a starting point for maximizing the copula log-likelihood. The individual negative binomial models have well-behaved log-likelihood functions and are relatively fast and straightforward to estimate by standard optimization techniques such as those presented in Section 3.2. Parameter estimates for the independent negative binomial models and the joint Gaussian copula model are presented in Table 2.

Table 2 Maximum simulated likelihood estimates of independent negative binomial (NB) models and joint Gaussian copula model with standard errors in parentheses

\begin{tabular}{|c|c|c|c|c|c|c|c|c|}
\hline & \multicolumn{4}{|c|}{ Independent NB Models } & \multicolumn{4}{|c|}{ Gaussian Copula Model } \\
\hline & $\mathrm{COM}$ & CHS & $\mathrm{CP}$ & IS & $\mathrm{COM}$ & CHS & $\mathrm{CP}$ & IS \\
\hline \multirow[t]{2}{*}{ Intercept } & 0.968 & -1.712 & -5.834 & -2.105 & 0.917 & -1.471 & -6.099 & -2.033 \\
\hline & $(0.993)$ & $(0.939)$ & (1.682) & $(0.568)$ & (1.040) & (0.986) & (1.645) & (0.628) \\
\hline \multirow[t]{2}{*}{$\ln (S A L E S)$} & -0.297 & -0.202 & 0.084 & -0.190 & -0.285 & -0.194 & 0.242 & -0.181 \\
\hline & $(0.254)$ & $(0.233)$ & $(0.423)$ & $(0.122)$ & $(0.270)$ & $(0.247)$ & $(0.417)$ & $(0.128)$ \\
\hline \multirow[t]{2}{*}{$\ln (W F)$} & 0.763 & 0.353 & 0.273 & 0.218 & 0.759 & 0.319 & 0.085 & 0.219 \\
\hline & $(0.210)$ & (0.194) & $(0.378)$ & $(0.140)$ & $(0.222)$ & $(0.203)$ & $(0.376)$ & (0.147) \\
\hline \multirow[t]{2}{*}{$\ln (R D C)$} & 0.081 & 0.611 & 0.717 & 0.631 & 0.078 & 0.580 & 0.665 & 0.608 \\
\hline & $(0.122)$ & $(0.091)$ & $(0.120)$ & $(0.080)$ & (0.128) & $(0.105)$ & $(0.148)$ & (0.089) \\
\hline \multirow[t]{2}{*}{$\ln \left(\alpha_{j}\right)$} & -0.174 & -0.017 & -0.564 & -0.464 & -0.184 & 0.026 & -0.563 & -0.451 \\
\hline & (0.090) & $(0.091)$ & $(0.131)$ & $(0.110)$ & (0.101) & (0.098) & $(0.150)$ & (0.119) \\
\hline \multirow{8}{*}{$\Omega$} & & & & & 1.000 & & & \\
\hline & & & & & $(0.000)$ & & & \\
\hline & & & & & 0.072 & 1.000 & & \\
\hline & & & & & $(0.070)$ & $(0.000)$ & & \\
\hline & & & & & 0.119 & 0.313 & 1.000 & \\
\hline & & & & & $(0.075)$ & $(0.053)$ & $(0.000)$ & \\
\hline & & & & & -0.080 & 0.225 & 0.115 & 1.000 \\
\hline & & & & & $(0.074)$ & $(0.063)$ & $(0.080)$ & $(0.000)$ \\
\hline
\end{tabular}

The results in Table 2 largely accord with economic theory. Of particular interest is the fact that in all cases the coefficients on $\ln (R D C)$ are positive, and for CHS, CP, and IS, they are also economically and statistically significant. Specifically, those point estimates are relatively large in magnitude and lie more than 1.96 standard errors away from zero, which is the 5\% critical value for a two-sided test under asymptotic normality. This indicates that innovation in those categories is capital-intensive and the stock of R\&D capital is a key determinant of patenting activity. The results also suggest that, all else being equal, the introduction of patents tends to be done by large firms, as measured by the size of the company workforce $\ln (W F)$. The coefficient on that variable in the communications category is large and statistically significant, whereas in the other three categories the estimates are positive but not significant at the customary significance levels. Interestingly, and 
perhaps counter-intuitively, the coefficients on $\ln (S A L E S)$ in these categories are predominantly negative (with the exception of computer peripherals), and none are statistically significant. To explain this puzzling finding, economists have proposed a rationalization that has to do with signaling in the presence of asymmetric information. In particular, firms that do not have steady sales revenue such as start-ups that have yet to establish a reliable customer base, are often cash constrained and may have to demonstrate their creditworthiness to potential lenders such as venture capitalists, banks, and individual investors in order to obtain loans. One way for such firms to exhibit their research innovations and overall productivity is to register patents. In this case patents serve a dual role - they protect the firm's innovations from infringement and also send a positive signal to potential outside stakeholders. In contrast, firms that have more reliable sources of revenue due to higher sales have lower incentives to patent their innovations and may instead opt to protect their intellectual property in other ways (e.g. by keeping trade secrets, entering into exclusive agreements with potential users of their technology, etc.). These considerations are especially relevant in the computers \& instruments industry, where patents have short life cycles and can often be circumvented by competitors who "innovate around" registered research advances.

Table 2 also illustrates that over-dispersion is a common feature of all four data series, as demonstrated by the low estimates of $\left\{\ln \left(\alpha_{j}\right)\right\}$ across all categories in both the copula and univariate regression models. As a consequence, allowing for over-dispersion by considering a negative binomial specification, as opposed to estimating a Poisson model, appears well justified.

In Table 2, the estimated dependence matrix $\Omega$ in the Gaussian copula model reveals interesting complementarities among patent categories and supports the case for joint modeling and estimation. Specifically, the estimates suggest that patents in the computer hardware \& software category are highly correlated with counts in the computer peripherals and information storage categories, while the correlation between patents in the communications category are relatively mildly correlated with those in the remaining categories. To test formally for the relevance of the copula correlation structure in this context, one can use the likelihood ratio and Lagrange multiplier tests. The log-likelihood for the restricted model (the independent negative binomial specification) is $L_{R}=-4050.06$ and for the unrestricted model (Gaussian copula model), it is $L_{U}=-4020.98$, leading to a likelihood ratio test statistic $-2\left(L_{R}-L_{U}\right)=58.16$. This test statistic has a $\chi^{2}$ distribution with 6 degrees of freedom (equal to the number of off-diagonal elements in $\Omega$ ) and a $5 \%$ critical value of 12.59 , suggesting that the data strongly reject the restricted (independent negative binomial) specification. The Lagrange multiplier test statistic is constructed from the gradient $g_{R}=\partial \ln f\left(y \mid \psi_{R}\right) / \partial \psi$ and curvature $B_{R}=\sum_{i=1}^{n} \frac{\partial \ln f\left(y_{i} \mid \psi_{R}\right)}{\partial \psi} \frac{\partial \ln f\left(y_{i} \mid \psi_{R}\right)}{\partial \psi^{\prime}}$ of the log-likelihood function of the Gaussian copula model, both evaluated at the restricted maximum likelihood estimate $\psi_{R}$. Note that this corresponds to the case when $\Omega$ is an identity matrix and the Gaussian copula model is equivalent to fitting the four negative binomial models separately (in fact, these are the starting values we use in optimizing the copula log-likelihood). The Lagrange multiplier test statis- 


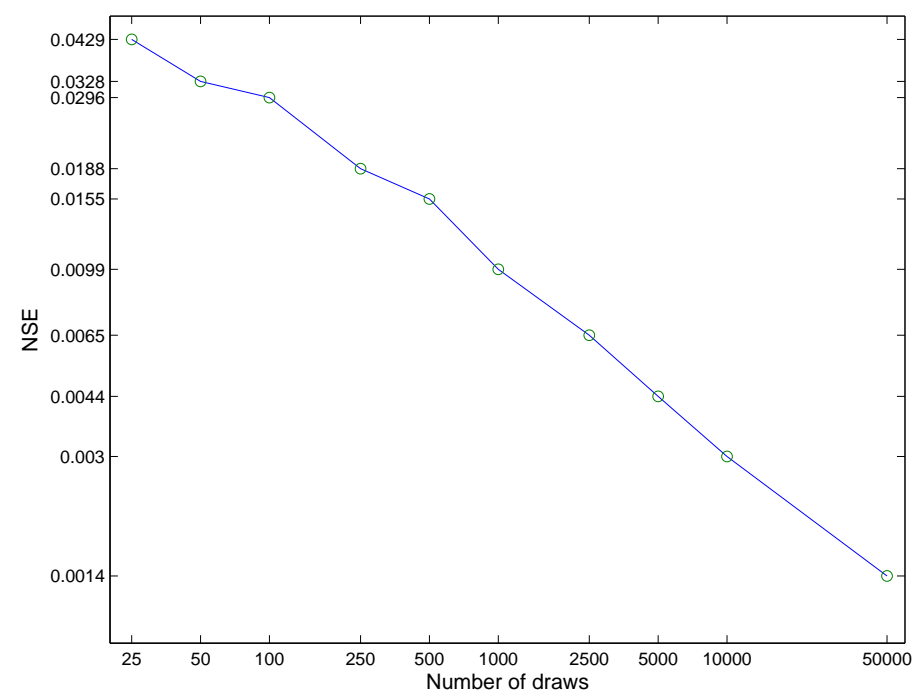

Fig. 2 Numerical standard errors (NSE) of the log-likelihood estimate as a function of the MCMC sample size in the CRT method (the axes, but not the values, are on the logarithmic scale)

tic $L M=\left\{g_{R}^{\prime}\left(B_{R}\right)^{-1} g_{R}\right\}=64.58$ has the same asymptotic $\chi^{2}$ distribution as the likelihood ratio statistic and also leads to strong rejection of the restricted model.

The parameter estimates and hypothesis tests presented above are based on maximizing an MCMC-based estimate of the log-likelihood function because that function is analytically intractable. However, because the variability intrinsic in simulation-based estimation can affect the reliability of the results, it is important to examine the extent to which the point estimates, standard errors, and test statistics are affected by the performance of the simulated likelihood algorithm. In Figure 2, we have plotted the numerical standard error of the estimated log-likelihood ordinate $\ln \hat{f}(y \mid \psi)$ as a function of the simulation sample size $G$ used in constructing the average in (13). The numerical standard error gives a measure of the variability of the estimated log-likelihood ordinate for fixed $y$ and $\psi$ if the simulation necessary to evaluate $\ln f(y \mid \psi)$ were to be repeated using a new Markov chain. The Figure demonstrates that the simulated likelihood algorithm performs very well even with few MCMC draws, the precision of estimating $\ln \hat{f}(y \mid \psi)$ is sufficient for producing reliable parameter estimates and hypothesis tests. The low variability of $\ln \hat{f}(y \mid \psi)$ in our example is especially impressive because the numerical standard errors are obtained as the square root of the sum of variances of the $n=498$ individual log-likelihood contributions. To be cautious, we have also verified the validity of the point estimates by initializing the algorithm at different starting values and also by estimating the model by Bayesian simulation methods similar to those proposed in [10] and [14], which do not rely on maximizing the log-likelihood.

At the end of Section 3.2, we discussed the possibility that in maximum simulated likelihood estimation the standard errors may be affected by simulation noise. To 


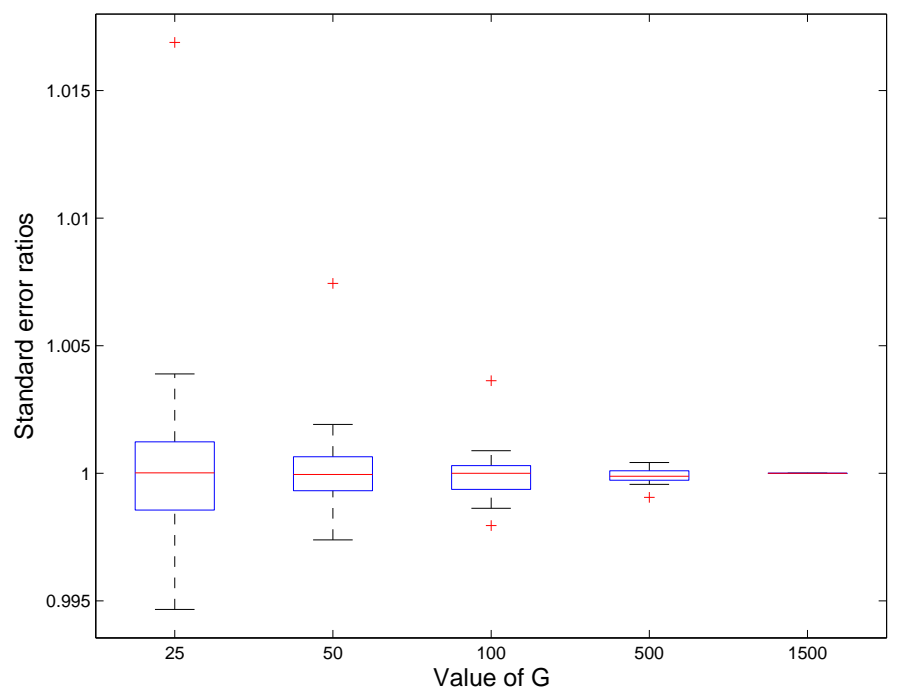

Fig. 3 Boxplots of the ratios of parameter standard errors estimated for each MCMC sample size setting $G$ relative to those for $G=1500$; the lines in the boxes mark the quartiles, the whiskers extend to values within 1.5 times the interquartile range, and outliers are displayed by " + "

examine the extent to which variability in the log-likelihood estimate translates to downward biases in the standard errors of the parameter estimates, we compute the standard errors across several settings of the simulation size $G$, namely $G \in$ $\{25,50,100,500,1500\}$. We then compare the behavior of the standard errors for lower values of $G$ relative to those for large $G$. Figure 3 presents boxplots of the ratios of the parameter standard errors estimated for each setting of $G$ relative to those at the highest value $G=1500$. The results suggest that while at lower values of $G$ the standard error estimates are somewhat more volatile than at $G=1500$, neither the volatility nor the possible downward bias in the estimates represents a significant concern. Because the CRT method produces very efficient estimates of the log-likelihood ordinate, such issues are not problematic even with small MCMC samples, although in practice $G$ should be set conservatively high, subject to one's computational budget.

\section{Concluding Remarks}

This chapter has discussed techniques for obtaining maximum simulated likelihood estimates in the context of models for discrete data, where the likelihood function is obtained by MCMC simulation methods. These methods provide continuous and differentiable estimates that enable the application of widely used derivative-based techniques for obtaining parameter standard errors and test statistics. Because we 
are maximizing a log-likelihood function, we rely on the BHHH outer product of gradients method to simplify and speed up the computation of the Hessian matrix of the log-likelihood. The methodology is applied in a study of the joint behavior of four categories of U.S. technology patents using a Gaussian copula model for multivariate count data. The results support the case for joint modeling and estimation of the patent categories and suggest that the estimation techniques perform very well in practice. Additionally, the CRT estimates of the log-likelihood function are very efficient and produce reliable parameter estimates, standard errors, and hypothesis test statistics, mitigating any potential problems (discussed at the end of Section 3.2) that could arise due to maximizing a simulation-based estimate of the log-likelihood function.

We note that the simulated likelihood methods discussed here can be applied in optimization algorithms that do not require differentiation, for example in simulated annealing and metaheuristic algorithms which are carefully examined and summarized in [22]. At present, however, due to the computational intensity of evaluating the log-likelihood function at each value of the parameters, algorithms that require numerous evaluations of the objective function can be very time consuming, especially if standard errors have to be computed by bootstrapping. Nonetheless, the application of such algorithms is an important new frontier in maximum simulated likelihood estimation.

\section{References}

1. Berndt, E., B. Hall, R. Hall, and J. Hausman (1974), "Estimation and Inference in Nonlinear Structural Models," Annals of Economic and Social Measurement, 3, 653-665.

2. Cameron, A. C. and P. K. Trivedi (1998), Regression Analysis of Count Data. Cambridge University Press.

3. Chib, S. (1995), "Marginal Likelihood from the Gibbs Output," Journal of the American Statistical Association, 90, 1313-1321.

4. Chib, S. and E. Greenberg (1996), "Markov Chain Monte Carlo Simulation Methods in Econometrics," Econometric Theory, 12, 409-431.

5. Geweke, J. (1991), "Efficient Simulation from the Multivariate Normal and Student-t Distributions Subject to Linear Constraints," in Computing Science and Statistics, ed. E. M. Keramidas, Proceedings of the Twenty-Third Symposium on the Interface, pp. 571-578, Fairfax: Interface Foundation of North America, Inc.

6. Greene, W. (2008), "Functional forms for the negative binomial model for count data," Economics Letters, 99, 585-590.

7. Hall, B. H. (1990), "The Manufacturing Sector Master File: 1959-1987," NBER Working paper 3366.

8. Jeliazkov, I. and E. H. Lee (2010), "MCMC Perspectives on Simulated Likelihood Estimation," Advances in Econometrics: Maximum Simulated Likelihood, 26, 3-39.

9. Joe, H. (1997), Multivariate Models and Dependence Concepts. London: Chapman and Hall.

10. Lee, E.H. (2010), Essays on MCMC Estimation, Ph.D. Thesis, University of California, Irvine.

11. Mairesse, J. and B. H. Hall (1996), "Estimating the Productivity of Research and Development: An Exploration of GMM Methods Using Data on French and United States Manufacturing Firms," NBER Working paper 5501. 
12. McFadden, D. and K. Train (2000), "Mixed MNL Models for Discrete Response," Journal of Applied Econometrics, 15, 447-470.

13. Nelsen, R. B. (1999), An Introduction to Copulas. New York: Springer-Verlag.

14. Pitt, M., D. Chan, and R. Kohn (2006), "Efficient Bayesian Inference for Gaussian Copula Regression Models," Biometrika, 93, 537-554.

15. Ritter, C. and M. A. Tanner (1992), "Facilitating the Gibbs Sampler: The Gibbs Stopper and the Griddy-Gibbs Sampler," Journal of the American Statistical Association, 87, 861-868.

16. Robert, C. P. (1995), "Simulation of Truncated Normal Variables," Statistics and Computing, $5,121-125$.

17. Sklar, A. (1959), "Fonctions de repartition 'a n dimensions et leurs marges," Publications de l'Institute de Statistique de l'Université de Paris, 8, 229-231.

18. Tierney, L. (1994), "Markov Chains for Exploring Posterior Distributions," Annals of Statistics, 22, 1701-1761.

19. Train, K. (2003), Discrete Choice Methods with Simulation. Cambridge: Cambridge University Press.

20. Trivedi, P. K., and D. M. Zimmer (2005), "Copula Modeling: An Introduction for Practitioners," Foundations and Trends in Econometrics, 1, 1-111.

21. Winkelmann, R. (2008), Econometric Analysis of Count Data, 5 edition. Berlin: Springer.

22. Yang, X.-S. (2010), Engineering Optimization: An Introduction with Metaheuristic Applications. Hoboken, New Jersey: John Wiley \& Sons. 